\title{
Measurement of Soret coefficients in a ternary mixture of toluene-methanol-cyclohexane in convection-free environment
}

\author{
A. Mialdun, ${ }^{1, a)}$ I. Ryzhkov, ${ }^{2}$ O. Khlybov, ${ }^{3}$ T. Lyubimova, ${ }^{3}$ and V. Shevtsova ${ }^{1, b)}$ \\ 1) MRC, CP165/62, Université Libre de Bruxelles, Av. F.D. Roosevelt, 50, B-1050, Brussels, \\ Belgium \\ ${ }^{2)}$ Institute of Computational Modelling SB RAS, Krasnoyarsk, Russia \\ ${ }^{3)}$ Institute of Continuous Media Mechanics UB RAS, Perm, Russia
}

\begin{abstract}
We report on the measurement of Soret $\left(S_{T}\right)$ coefficients in the ternary system toluene (T)-methanol (M) -cyclohexane $(\mathrm{Ch})$ onboard the International Space Station (ISS) in the experiment SODI/DCMIX . Nine experiments were conducted in the range of mean temperatures between $298.15 \mathrm{~K}$ and $306.15 \mathrm{~K}$ in the mixture with composition $0.62(\mathrm{~T})-0.31(\mathrm{M})-0.07(\mathrm{Ch})$ in mass fractions. A linear dependence of the Soret coefficients on temperature was established for the first time in ternary mixtures. It has also been found that, over considered range of mean temperatures, the Soret coefficients of toluene are small and positive, while the Soret coefficients for methanol are negative and, at least, two times larger. The present work also presents a comprehensive study of possible methodologies to process raw data from the Soret experiment in ternary mixtures. All the experiments were processed by seven different schemes and two of them were identified as the most reliable. We also investigate the error propagation and explain the reasons for discrepancy of the results obtained by different schemes.
\end{abstract}

Keywords: Soret effect, liquid mixtures, microgravity, optical technique

\section{INTRODUCTION}

There are many important processes in nature and technology, where diffusion and thermodiffusion phenomena play a decisive role. Thermodiffusion (also known as thermal diffusion or Soret effect) refers to a transport mechanism in which temperature gradients cause mass transfer in mixtures. The composition of underground hydrocarbon reservoirs is significantly affected by diffusion as well as by the Soret effect due to the presence of a geothermal gradient ${ }^{1,2}$. On the microscale, thermodiffusion allows a controlled manipulation of small particles and molecules, e.g., directing DNA molecules and polystyrene beads drift along a temperature gradient ${ }^{3}$. In polymer blends ${ }^{4}$, phase separation by thermodiffusion has potential application for nanotechnology in diverse fields, ranging from bioactive patterns to polymer electronics.

The experimental techniques for measurement of Soret coefficients in liquid binary mixtures are well established, and they are well presented in recent reviews ${ }^{5,6}$. During the past decade, however, the interest of science moves towards ternary mixtures that can be seen as prototypes for truly multicomponent mixtures.

The complexity increases significantly when going from binaries to ternaries. If we denote the mass fraction of component $i$ by $c_{i}$, i.e., $\left(c_{1}+c_{2}+c_{3}=1\right)$ then in ternary mixture the diffusive fluxes of the independent components $(i=1,2)$ will be written as

$$
\begin{aligned}
& j_{1}=-\rho\left(D_{11} \nabla c_{1}+D_{12} \nabla c_{2}+D_{T, 1}^{\prime} \nabla T\right) \\
& j_{2}=-\rho\left(D_{21} \nabla c_{1}+D_{22} \nabla c_{2}+D_{T, 2}^{\prime} \nabla T\right)
\end{aligned}
$$

\footnotetext{
a) Electronic mail: amialdun@ulb.ac.be

b) Electronic mail: vshev@ulb.ac.be
}

where $D_{i k}$ are the mass-based diffusion coefficients and $D_{T i}^{\prime}$ are the thermal diffusion coefficients (here $D_{T, i}^{\prime}=$ $\left.c_{i}\left(1-c_{i}\right) D_{T, i}\right)$. In the stationary state the diffusion fluxes vanish $\left(j_{i}=0\right)$ and the concentration gradients are proportional to the temperature gradient

$$
\nabla c_{i}=-S_{T, i}^{\prime} \nabla T, \quad i=1,2,
$$

where the Soret coefficients $\left(S_{T, i}^{\prime}\right)$ are determined as

$$
S_{T, i}^{\prime}=\sum_{k=1}^{2}\left(\mathbf{D}^{-\mathbf{1}}\right)_{i k} D_{T k}^{\prime}, \quad \sum_{i=1}^{3} S_{T, i}^{\prime}=0
$$

$\left(\mathbf{D}^{-\mathbf{1}}\right)_{i k}$ denotes an element of the inverse diffusion matrix. Thus, six unknown quantities, four diffusion and two thermodiffusion coefficients, have to be determined in the course of experiments.

The values of diffusion coefficients in ternary mixtures depend on the order of the components as well as on the frame of reference for which the diffusive fluxes are written. Accordingly, the value of the sequential Soret coefficient $\left(S_{T, i}^{\prime}, \mathrm{i}=1,2,3\right)$ is assigned to a specific component. We adopted a hydrodynamic approach to the numbering of components, which corresponds to a decreasing order of density: toluene (component 1), methanol (component 2) and cyclohexane (component 3).

The first laboratory measurements to determine these coefficients on truly ternary systems by different techniques displayed significant divergence of the results ${ }^{7,8}$. Note that the methods applied in the above works ${ }^{7,8}$ are very different from one another, even by the basic principle they use, and they provide either $S_{T, i}^{\prime}$ or $D_{T, i}^{\prime}$. A dedicated approach has been developed ${ }^{9}$ to cross-check conflicting measured values, and it showed that thermodiffusion coefficients are extremely sensitive to the precise values of mass diffusion coefficients and optical contrast 
factors. In addition, ternary systems are further complicated by the fact that the sign of the Soret coefficients of the various components can be different and this can lead to convective instability which affects the results.

In the framework of the international DCMIX project, scientists expect to obtain reliable benchmark results on the ISS for the validation and calibration of present and future ground based measurements. The SODI (Selectable Optical Diagnostic) instrument operating onboard the ISS is equipped with a two-wavelength interferometer which enables to measure Soret and diffusion coefficients in ternary mixtures. A significant step was achieved by IVIDIL (Influence VIbration on DIffusion in Liquids), the first experiment inside the SODI, which examined a binary solution, and had confirmed that the daily onboard environment of the ISS does not perturb diffusion-controlled experiments ${ }^{10}$.

The DCMIX project is divided into four campaigns that focus on different ternary mixtures. The SODI instrument is kept on the ISS and for each campaign only a new cell array is delivered. The cell array consists of five primary cells filled with ternary mixture and one companion cell with binary mixture.

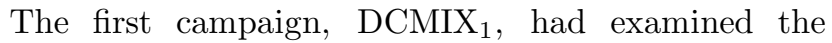
ternary mixture composed of tetralin (THN), isobutylbenzene (IBB) and dodecane (nC12). This mixture is already actively used by petroleum industry for their numerical modeling as it includes molecules of different families (polycyclic, alkane, aromatic). Another advantage of this system is that the Soret coefficients of denser components are positive and it provides the possibility of performing comparative measurements in the gravity field. The comparison of the results obtained on orbit with the laboratory measurements displayed favorable agreement, while the reasons for the observed discrepancy were thoroughly analyzed ${ }^{11}$. One of the insights obtained from this comparison concerns the propagation of errors related to the poor optical properties of the mixture. Hereinafter this issue will be discussed in more detail.

The second system, DCMIX 2 , is composed by toluene, methanol and cyclohexane. This highly non-ideal system is of particular interest due to the existence of a miscibility gap and a large region of compositions with negative Soret coefficients. Its investigation in ground based experiments is significantly more complex than the DCMIX $_{1}$ mixtures due to convective instabilities.

The DCMIX $_{3}$ system is composed by water, ethanol and triethylene glycol. Its experiment on the ISS was finalized recently and scientists have started processing the results.

The last campaign, DCMIX $_{4}$, will include systems of different origins: three cells with the same system as DCMIX $_{2}$, one cell with a nanofluid ${ }^{12}$ (tetralin, toluene, fullerene) and two cells with mixtures containing a polymer $^{13}$; ternary (polystyrene, toluene, n-hexane) and binary (polystyrene, toluene).

The subject of the present study is the DCMIX $_{2}$ system which was examined on the ISS during seven weeks
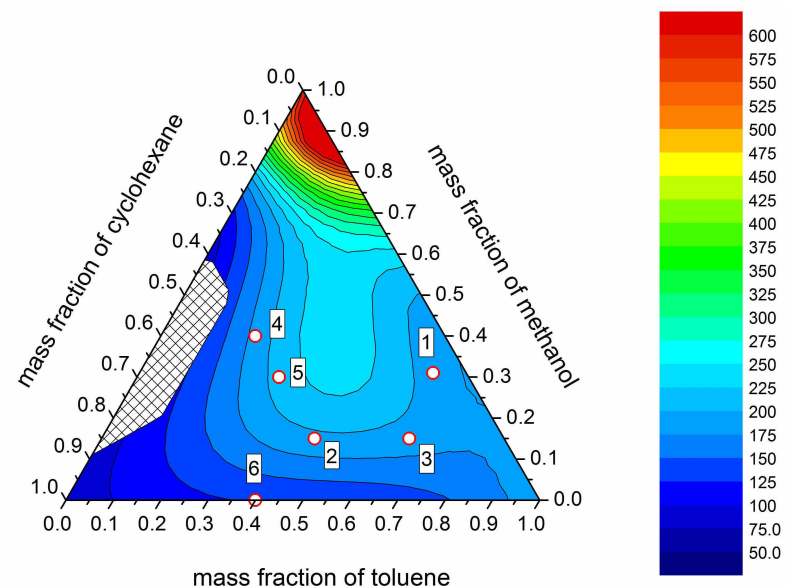

FIG. 1. The compositions of the DCMIX $_{2}$ ternary system toluene-methanol-cyclohexane in microgravity experiments are shown by circles. The colors indicate condition number of the contrast factor matrix of the system. The hatched area indicates a miscibility gap.

in December 2013-January 2014. One of the questions to be answered in the course of the experiment is how transport coefficients diverge when approaching the miscibility region (binodal). Five cells were filled with ternary mixtures of different compositions at various distance from the binodal curve as shown in Fig. 1.

In this work, we explicitly investigate the mixture in cell \#1 with the composition $0.62 / 0.31 / 0.07$ in mass fractions. There are several motivations to process the mixture in this cell first. Preliminary ground tests ${ }^{14}$ indicated that among the five ternary systems in the DCMIX $_{2}$ only this one can be potentially studied in ground laboratories. Thus it is a suitable candidate for future benchmarking and, respectively, there is a need to find out the best approach to process raw data. The measurements in the cell were reproducible and, unlike other cells, no permeation problem ${ }^{15}$ occurred on long time scale.

The present paper is organized as follows. In section II, we describe the SODI experiment, the choice of mixture compositions and the experimental scenario. The different steps of image processing are shortly described in section III, followed by the selection of the best processing schemes. The experimental results on Soret separation onboard the ISS obtained by the selected schemes are presented in section IV. Finally, conclusions are drawn. More details of image processing is given in Supplementary material.

\section{EXPERIMENTAL}

\section{A. SODI instrument and flight campaign}

The onboard instrument used in the present work has been extensively described already in literature ${ }^{16-18}$ and 
TABLE I. List of liquid mixtures settled in the DCMIX $_{2}$ cell array. Compositions are given in mass fractions.

\begin{tabular}{cccc}
\hline cell_ID & $\begin{array}{c}\text { Toluene } \\
\mathrm{kg} \mathrm{kg}^{-1}\end{array}$ & $\begin{array}{c}\text { Methanol } \\
\mathrm{kg} \mathrm{kg}^{-1}\end{array}$ & $\begin{array}{c}\text { Chyclohexane } \\
\mathrm{kg} \mathrm{kg}^{-1}\end{array}$ \\
\hline cell \#0 & 0.40 & $\ldots$ & 0.60 \\
cell \#1 & 0.62 & 0.31 & 0.07 \\
cell \#2 & 0.45 & 0.15 & 0.40 \\
cell \#3 & 0.65 & 0.15 & 0.20 \\
cell \#4 & 0.20 & 0.40 & 0.40 \\
cell \#5 & 0.30 & 0.30 & 0.40 \\
\hline
\end{tabular}

here we will only outline its key features.

The optical design of the SODI is based on the concept of a Mach-Zehnder interferometer and includes two optical benches: moving and fixed. The cell array is fixed and the moving optical bench allows positioning the optics in front of and behind the studied cell. This optical system is equipped with two laser diodes of $\lambda_{1}=670 \mathrm{~nm}$ and $\lambda_{2}=935 \mathrm{~nm}$ and, thus, it allows two-wavelengths optical digital interferometry (ODI) to resolve two independent concentrations in ternary mixtures ${ }^{18}$. A fixed optical bench with third laser diode $(\lambda=670 \mathrm{~nm})$ is used for the cell filled with the binary mixture ${ }^{19}$. These benches with electronics, connectors, storage, constitute the SODI facility. The experiment cell array flown in the SODI, consists of six small cells, as shown in Fig. 2. Each cell is a transparent rectangular glass frame with inner dimensions of $L \times W \times H=10 \mathrm{~mm} \times 10 \mathrm{~mm} \times 5 \mathrm{~mm}$. The cell design is outlined in Fig. 3. The top and bottom are closed with $2 \mathrm{~mm}$ protruding end caps of nickel-plated copper. These protrusions and a compensation volume for thermal expansion are responsible for a deviation of the temperature field from linearity in the vertical direction, particularly in the corners of the cell (see Fig.3 in Ref. $\left.^{20}\right)$.

The schedule of the experimental campaign DCMIX $_{2}$ was as follows: the cells were filled 35 days before the launch to the ISS on November 26, 2013. Five days after upload, the cell array was installed in the SODI instrument, it passed optical check-out tests, and the scientific experiments started on December 1, 2013. Last experimental run has been completed on January 23, 2014.

While the orbital experiment was being conducted, the SODI engineering model, which is an exact replica of the flight one, was readily available on the ground and used for testing the impact of the last minutes changes in the experimental program.

\section{B. Liquids mixture and selection of experimental points}

Chemicals used for the mixture preparation were: toluene, $99.85 \%$ for residue analysis, of Acros Organics; methanol, 99.99\% analytical reagent grade, of Fisher Chemical; and cyclohexane, $99.97 \%$ analytical reagent grade, of Fisher Chemical. The chemicals were used without further purification. The ternary mixtures for

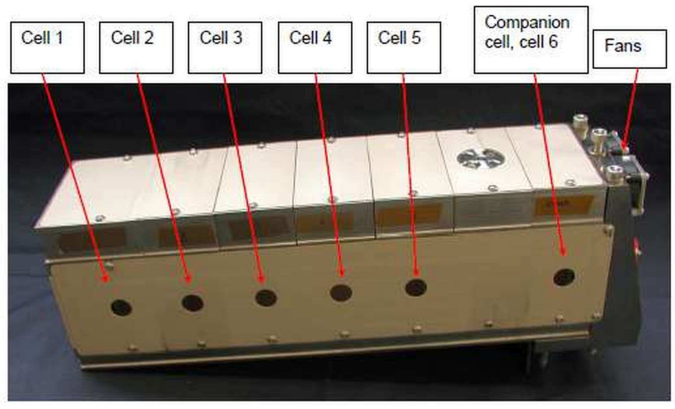

FIG. 2. Cell array of DCMIX experiments. The five cells filled with ternary mixtures are called as the primary cells and one cell filled with a binary mixture is called as the companion cell.

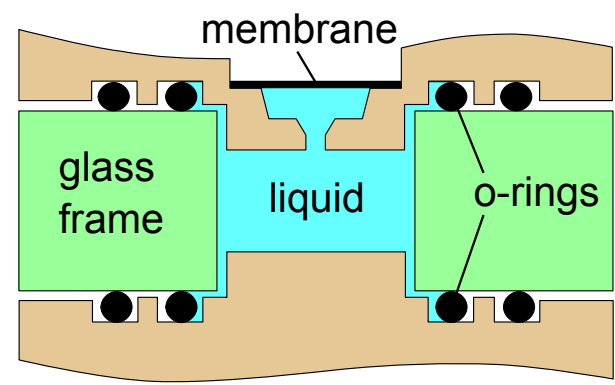

FIG. 3. Schematic of the central part of the experiment cell.

the microgravity experiment were prepared by weighing. After preparation and thorough mixing, the mixtures were degassed by the freeze-melting technique and stored in tightly closed bottles until the filling of the cells. Prior delivery to QinetiQ Space N.V. company, where the cells were filled, the mixtures were placed into syringes (Hamilton, Gas-tight, $10 \mathrm{ml}$ ) avoiding contact with air.

The compositions of the ternary mixture for DCMIX $_{2}$ were selected following several criteria. The most important one is related to the optical properties of the mixture. The separation of the components caused by the Soret effect was monitored by measuring the temperature and concentration fields inside the cell via a twowavelength interferometer. The thermal and concentration contributions can be separated, see section III A 3 . In what concerns the concentration field, the inputs into the refractive index at each wavelength $\lambda_{i}$ include contributions from two components,

$$
\begin{aligned}
& n_{1}^{\prime}(z, t)=R_{11} c_{1}^{\prime}(z, t)+R_{12} c_{2}^{\prime}(z, t), \\
& n_{2}^{\prime}(z, t)=R_{21} c_{1}^{\prime}(z, t)+R_{22} c_{2}^{\prime}(z, t),
\end{aligned}
$$

where prime denotes the deviation of a quantity from its average value, e.g., $n^{\prime}(x, z)=n(x, z)-n_{0}$ and $R_{i j}=$ $\left(\partial n_{i} / \partial c_{j}\right)_{p, T, c_{i}, i \neq j}$ are the optical contrast factors.

Here $n_{i}^{\prime}(z, t)$ are experimentally measured distributions of the refractive index while the concentration profile has to be determined. An algorithm for solving systems of 
TABLE II. Physical properties of the mixture toluene-methanol-cyclohexane with concentration of $0.62 / 0.31 / 0.07$ mass fraction at $T_{0}=298.15 \mathrm{~K}$ : density $\rho$, thermal $\beta_{T}$ and solutal $\beta_{C}$ expansions, kinematic viscosity $\nu$, thermal diffusivity $\chi$

\begin{tabular}{|c|c|c|c|c|c|c|}
\hline $\begin{array}{c}\rho \\
k g / m^{3}\end{array}$ & $\begin{array}{c}\beta_{T} / 10^{-3} \\
\mathrm{~K}^{-1}\end{array}$ & $\beta_{c_{1}}$ & $\beta_{c_{2}}$ & $\begin{array}{c}\mu / 10^{-3} \\
P a \cdot s\end{array}$ & $\begin{array}{c}\nu / 10^{-6} \\
m^{2} / s\end{array}$ & $\begin{array}{c}\chi / 10^{-7} \\
m^{2} / s\end{array}$ \\
\hline $829.99^{\mathrm{a}}$ & $1.16^{\mathrm{a}}$ & -0.140 & -0.048 & $0.56^{\mathrm{a}}$ & $0.67^{\mathrm{a}}$ & 0.94 \\
\hline
\end{tabular}

a data from ref. ${ }^{21}$

c data from ref. ${ }^{22}$

TABLE III. Characteristic viscous $\left(\tau_{v i s}=H^{2} / \nu\right)$, thermal $\left(\tau_{t h}=H^{2} / \chi\right)$ and diffusion $\left(\tau_{D}=H^{2} / \hat{D}_{2}\right)$ times when $H=0.005 \mathrm{~m}$; the corresponding Prandtl $\operatorname{Pr}=\nu / \chi$, and eigenvalues $\hat{D}_{i}$ of diffusion matrix.

\begin{tabular}{|c|c|c|c|c|c|}
\hline$\tau_{v i s}$ & $\tau_{t h}$ & $\tau_{D} / 10^{4}$ & $P r$ & $\hat{D}_{1} / 10^{-9}$ & $\hat{D}_{2} / 10^{-9}$ \\
\hline $\mathrm{S}$ & $\mathrm{S}$ & $\mathrm{S}$ & & $\mathrm{m}^{2} / \mathrm{s}$ & $\mathrm{m}^{2} / \mathrm{s}$ \\
\hline$\overline{37.3}$ & 266 & 3.42 & 7.1 & $2.05^{\mathrm{a}}$ & $0.73^{\mathrm{a}}$ \\
\hline
\end{tabular}

linear equations (5)-(6) requires the inversion of the matrix of optical contrast $\mathbf{R}$ which can be ill-conditioned ${ }^{25}$. Before the orbital experiment, the refractive index of the mixture was measured at two available wavelengths $(670 \mathrm{~nm}$ and $925 \mathrm{~nm})$ for the entire concentration space, and then condition numbers were calculated ${ }^{24}$. Blue colors in Fig. 1 outline a region with favorable optical properties for experiments inside the SODI and the color scale indicates the value of the condition number. Correspondingly, the compositions for the experiment were selected in the region with the suitable condition number and as close as possible to the demixing zone. However, the preflight tests have shown that not all mixture compositions are compatible with seal materials ${ }^{14}$ and the originally selected composition for the state point \#1 was moved rather far from demixing zone. The compositions of the cells examined in microgravity experiment are given in Table I.

The physical and optical properties of the mixture \#1, examined in this study, are listed in Tables II-IV; as to the quantities which were not measured in this work, the source of data is indicated.

\section{Experimental procedure}

During the Soret experiment, two opposite surfaces of a cell were maintained at two different temperatures, so as to induce thermodiffusion inside the fluid contained therein. Experiments in each cell with ternary mixture were carried out in turn. Such an order of execution provided a time interval of several days between consecutive runs in the same cell, which is necessary to ensure
TABLE IV. Optical contrast factors for toluene-methanolcyclohexane mixture measured $^{24}$ at $T=298.15 \mathrm{~K}$ and the condition number $\mathcal{K}$ of the contrast factors matrix at cell \#1.

\begin{tabular}{llll}
\hline wavelength & $\left(\partial n / \partial c_{1}\right)$ & $\left(\partial n / \partial c_{2}\right)$ & $\mathcal{K}$ \\
\hline $670 \mathrm{~nm}$ & 0.079490 & -0.095686 & 80 \\
$925 \mathrm{~nm}$ & 0.075026 & -0.095039 & \\
\hline
\end{tabular}

TABLE V. List of analysed experimental runs conducted with the cell \#1.

\begin{tabular}{llcccc}
\hline Run & Date & $\begin{array}{c}T_{0} \\
(\mathrm{~K})\end{array}$ & $\begin{array}{c}\Delta T \\
(\mathrm{~K})\end{array}$ & Steps $^{\mathrm{a}}$ & $\begin{array}{c}\text { Duration }^{\mathrm{b}} \\
\text { (hour) }\end{array}$ \\
\hline Run_01 & 01-Dec-2013 & 298.15 & 5.0 & $\mathrm{~S}+\mathrm{D}$ & 10 \\
Run_06 & 07-Dec-2013 & 298.15 & 10.0 & $\mathrm{~S}+\mathrm{D}$ & 10 \\
Run_01bis & 10-Dec-2013 & 298.15 & 5.0 & $\mathrm{~S}+\mathrm{D}$ & 10 \\
Run_11 & 13-Dec-2013 & 301.15 & 5.0 & $\mathrm{~S}+\mathrm{D}$ & 10 \\
Run_16 & 26-Dec-2013 & 304.15 & 5.0 & $\mathrm{~S}+\mathrm{D}$ & 10 \\
Run_21 & 07-Jan-2014 & 298.15 & 5.0 & $\mathrm{~S}$ & 10 \\
Run_26 & 09-Jan-2014 & 295.15 & 5.0 & $\mathrm{~S}+\mathrm{D}$ & 10 \\
Run_32 & 16-Jan-2014 & 304.15 & 5.0 & $\mathrm{~S}$ & 15 \\
Run_33 & 23-Jan-2014 & 306.15 & 5.0 & $\mathrm{~S}+\mathrm{D}$ & 15 \\
\hline
\end{tabular}

a 'S' stands for the Soret step, and ' $D$ ' is for the diffusion step.

b Duration is the time prescribed for each of the steps.

an undoubted homogenization of the mixture after Soret separation.

All the runs conducted in the cell \#1 are listed in Table $\mathrm{V}$, where the main features of each run are outlined. The runs have been numbered sequentially by the order of execution, thus, there is mostly an increment of 5 in between the run identifiers for the same cell. The consecutive runs in the cell \#1 are separated in time by experiments in cells \#2-5.

As can be seen from Table V, the implemented set of runs covers a temperature range from 295.15 to $306.15 \mathrm{~K}$, including several repetitions at mean temperatures $T_{0}=$ 298.15 and $304.15 \mathrm{~K}$.

As a rule, experiments were carried out applying tem-

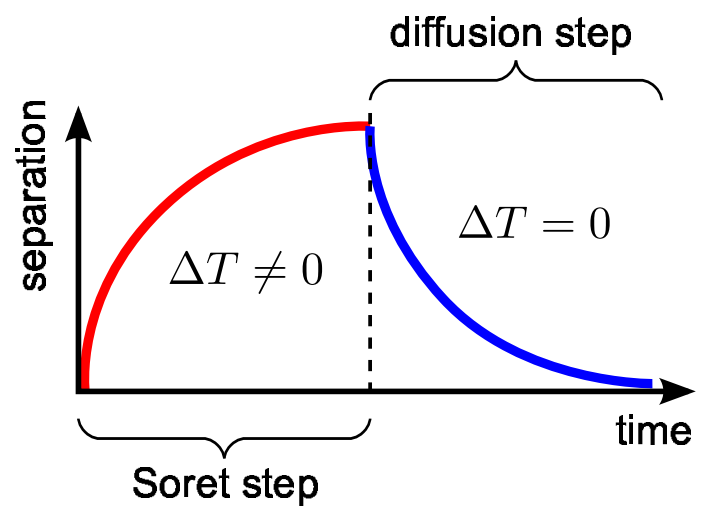

FIG. 4. Time-line of the DCMIX experiment: Soret and diffusion steps. 


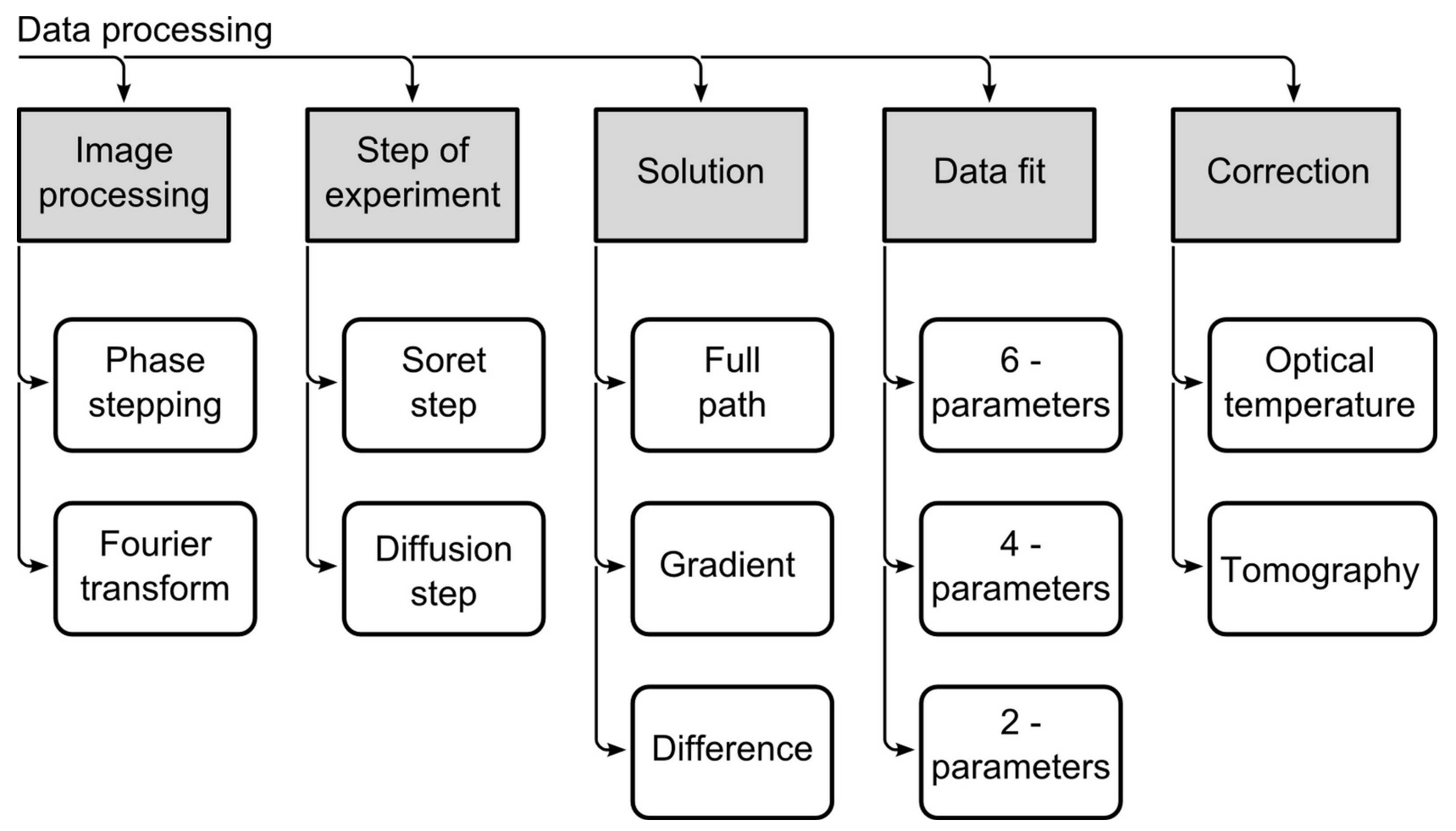

FIG. 5. Visual guidance on possible schemes for processing results. The shaded panels indicate the major successive steps and they correspond to the content of subsections in Section III. The possible executions of each step are indicated by non-shaded panels.

perature difference $\Delta T=5.0 \mathrm{~K}$. The created temperature gradient $d T / d z=1 \mathrm{~K} / \mathrm{mm}$ is considered as the optimal value according to our ground experiments. A larger value of $d T / d z$ may affect the linear relation, see Eq. (3), because the Soret coefficient is temperature dependent. A smaller value of $d T / d z$ may result in a too weak amplitude of the signal that leads to a larger uncertainty. In order to check the validity of the above assumptions, one experiment, Run_06, was carried out with $\Delta T=10.0 \mathrm{~K}$.

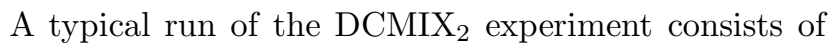
two main steps, Soret separation and diffusion relaxation, as outlined in Fig.3. Once the mixture is thermally stabilized, a temperature difference is imposed between opposite walls, and the separation of the mixture occurs due to thermal diffusion during an assigned time (the Soret step). After that, the temperature gradient is removed, the mixture becomes isothermal and gradually homogenized by diffusion (the Diffusion step). In order to save time, the monitored diffusion step was skipped in two runs (Run_21 and Run_32).

The durations of the above two steps in the DCMIX 2 were identical and have been chosen according to the expected diffusion time for each particular mixture. The diffusion time was estimated in advance using previously measured mass diffusion matrix ${ }^{23}$

$$
\tau_{D}=H^{2} / \min \left(\hat{D}_{i}\right)
$$

where eigenvalues $\hat{D}_{i}$ are defined as

$$
\hat{D}_{1,2}=\frac{D_{11}+D_{22} \pm \sqrt{\left(D_{11}-D_{22}\right)^{2}+4 D_{12} D_{21}}}{2}
$$

For the cell \#1 the determined eigenvalues are given in table III and, correspondingly, the duration of the experiment was fixed to $10 \mathrm{~h}$. In order to verify how close the system is to steady state, two experimental runs lasted longer by $50 \%$.

\section{DATA PROCESSING}

The format of data obtained from the DCMIX experiment is a set of interference patterns in the form of fringe images. Processing of the fringe images with the aim to obtain Soret/diffusion coefficients consists of several main steps:

- recover the continuous optical phase field and, subsequently, the map of refractive indices from interferograms;

- determine the transport coefficients from the map of refractive indices by fitting the experimental data to analytical solution;

- apply corrections to the original data in case it is somewhat distorted. 

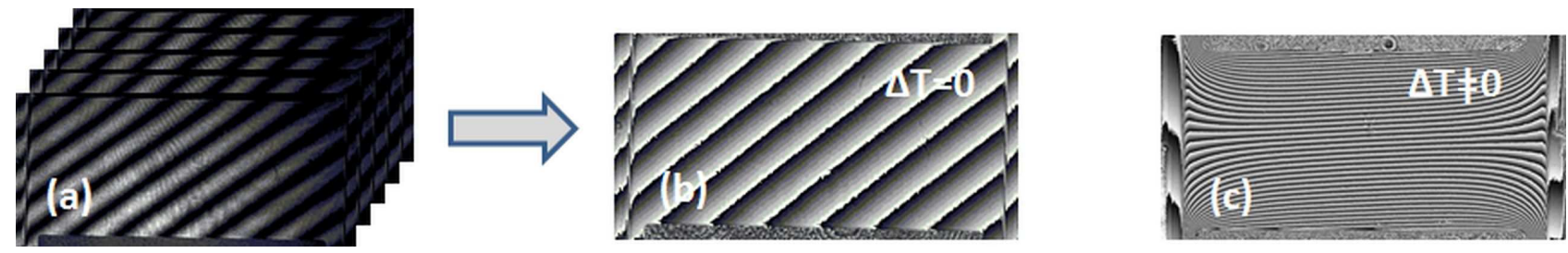

FIG. 6. Interferometric patterns. (a) five original fringe images; (b) combined fringe images into single phase image; (c) phase image after imposing the temperature difference $\Delta T$ across the cell

Each of these steps can be implemented in different ways, which offer a wide variety of approaches to data processing. In the course of benchmarking for the DCMIX $_{1}$ experiment, several groups have processed the same set of fringe images by different procedures ${ }^{11}$. The mass transfer coefficients, obtained by the different procedures, have revealed a noticeable dispersion. To address this problem, we begin our study by identifying the main sources of differences in data processing and analyzing their potential impact on intermediate and final results.

\section{A. Analysis of different processing stages}

Several sources, which can lead to the different final results, were identified and classified, each of them is considered separately in the following subsections and more details are given in Supplementary. A visual guide of the possible schemes of the results processing is given in Fig. 5. Each following subsection corresponds to the subsequent shaded panel in Fig. 5. Several steps are also used to process raw data for binary mixture. An analysis of all the DCMIX $_{2}$ experiments conducted in the cell with binary mixture Tol-Chex with concentration of $0.40 / 0.60$ mass fraction has confirmed the consistency of the results obtained in microgravity with those from ground laboratories ${ }^{19}$.

\section{Reconstruction of optical phase: phase-shift or Fourier transform}

Spatial reconstruction of optical phase from the fringe pattern can be implemented in different ways. The design of the interferometer onboard the ISS is based on the phase shifting technique. Five fringe images with constant phase-shift, created by changing the driving current on laser, were acquired to get one phase image ${ }^{16,18}$, as shown in Fig. 6(a)-(b). The optical system was aligned for wide fringe configuration because such alignment is favourable for the phase-shift processing. Resolution of imaging system is about 150 pixels $/ \mathrm{mm}$.

However, the density of fringes significantly increases when the temperature gradient is imposed across the cell, see Fig. 6(c) and this provides a possibility for the application of different techniques of picture processing. Two groups ${ }^{17,26}$ have demonstrated that the Fourier transform technique, which considers only one fringe image of a band of five, can also be used for the optical phase extraction.

Both of the above methods differ substantially in the basic principle, therefore, one can expect some difference in the optical phase obtained either with the phase shift or the Fourier transform approach. Note that even within the same methodology, the difference in results obtained by different researchers may arise due to the way of its implementation

\section{Steps of the experiment: Soret or diffusion}

Most of microgravity experiments consists of the two steps: the Soret and diffusion steps as shown in Fig 4. A spatial and temporal evolution of a concentration in each step is described by diffusion and thermodiffusion coefficients, thus, each step of the experiment is an independent source of the data. This concept is equally valid for binary and ternary mixtures. As an illustrative example, it is useful to write here a general form of the solution for a binary mixture. Then, the concentration evolution in a mixture can be written $\operatorname{as}^{27}$

$$
c(z, t)-c_{0}=\Delta c^{\circ} f(z, t, D),
$$

where $c_{0}$ is an initial (also average) concentration of a mixture, $\Delta c^{\circ}=\Delta c^{\text {st }}\left(D_{T}, D, \Delta T\right)$ is the concentration difference between hot and cold walls (the Soret separation) in steady-state. The dimensionless function $f(z, t, D)$ describes the spatial and temporal evolution of the concentration, and it includes the exponential term with diffusion coefficient as an exponent.

It is important to note that the representation of the function $f(z, t, D)$ for Soret and diffusion steps has minor difference, in the considered case is as simple as $f_{S}=$ $1-f_{D}$. This gives an idea that one cannot expect a difference between the results obtained at different steps of the same experimental run, but, as can be seen below, in practice, there is a noticeable difference ${ }^{26}$.

\section{Theoretical approach}

(a) Separation of thermal and compositional contributions 
In optical probing, the spatial concentration variation in the mixture is evaluated by measuring the change in the refractive index (optical phase). Since the Soret separation is a non-isothermal process, the refractive index variation appears as a sum of two contributions, thermal and compositional

$$
n^{\prime}(x, z)=\left(\frac{\partial n}{\partial T}\right)_{c, p} T^{\prime}(x, z)+\left(\frac{\partial n}{\partial c}\right)_{T, p} c^{\prime}(x, z),
$$

where prime denotes quantities normalized to their average value, e.g., $n^{\prime}(x, z)=n(x, z)-n_{0}$. Hereafter the subscripts for the derivatives of refractive index with temperature and concentration are omitted, assuming that all parameters are kept constant except the one by which the derivative is taken.

In liquid mixtures, the heat transfer by conduction occurs much faster than the mass transfer by diffusion. Accordingly, a classical approach for separation of the contributions relies on a large difference between thermal $\left(\tau_{t h}\right)$ and diffusion $\left(\tau_{D}\right)$ characteristic times, they are given in table III. A simplified treatment considers only the first term in right-hand side (RHS) of Eq. (9) for the short initial time $t<\tau_{t h}$ and only the second term in RHS of Eq. (9) for the time $t>\tau_{t h}$, respectively ${ }^{17}$. This approach is violated for small times, where the heat and mass transfer occur simultaneously, but can be used for preliminary rough evaluation.

In advanced approaches the contributions of concentration at earlier times $\left(t<\tau_{t h}\right)$ is taken into account either by use of a full direct numerical simulation of the thermal and diffusion processes ${ }^{28,29}$, or by applying special technical means for reducing the time of establishing the thermal gradient to a few seconds instead of minutes ${ }^{30}$, or by analytical evaluation of the separation during the transient thermal step, first proposed in Ref. ${ }^{31}$. In what concerns the DCMIX experiment, the latter approach is the only feasible one. Its detailed description can be found elsewhere ${ }^{18,26}$, and it is applied in the present work.

It is worth noting here that this problem of the separation of contributions arises only at the Soret step; the isothermal diffusion step does not contain the thermal contribution. Thus, using data either from Soret or diffusion step can lead to a difference in the final results, the comparison of the results will be discussed in Sec. IV.

(b) Type of analytical solution describing the mass transport

To recover the mass transport coefficients, the same spatial concentration distribution $c^{\prime}(x, z)$ can be related to a different analytical solution and, consequently, it can be treated in different ways. Here again, to illustrate the idea we use an example of binary mixture assuming that the mass transfer occurs in the z-direction only.

Three most widely used analytical solutions are: (a) the full path solution that includes all available data points, both in time and in space. The solution for the Soret step can be presented in a form of Eq. (8) where

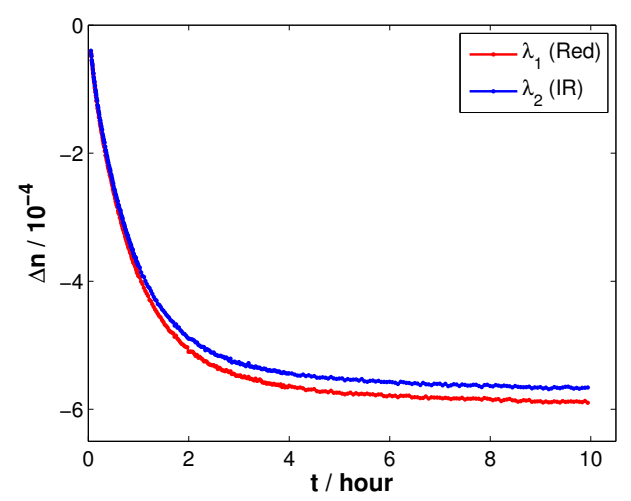

FIG. 7. The time evolution of the refractive index difference between two hot and cold walls during the Soret step measured at two wavelengths at Run_01, $\Delta n_{i}(t)=n_{i}^{\prime}(z=$ $H, t)-n_{i}^{\prime}(z=0, t)$.

the function $f(z, t, D)$ is written as

$$
f_{S}=\frac{1}{2}-\frac{z}{H}-\frac{4}{\pi^{2}} \sum_{n=1}^{\infty} \frac{1}{(2 n-1)^{2}} \cos \left(\frac{(2 n-1) \pi z}{H}\right) E_{2 n-1}
$$

with diffusion coefficient $D$ entering the exponential term

$$
E_{2 n-1}=\exp \left(-\frac{(2 n-1)^{2} \pi^{2}}{H^{2}} D t\right) .
$$

(b) the gradient solution (resembling an Optical Beam Deflection ${ }^{28}$ approach) describes the gradient of concentration at mid-height of the cell:

$$
\frac{\partial c}{\partial z} \frac{H}{\Delta c^{\mathrm{st}}}=1-\frac{4}{\pi} \sum_{n=1}^{\infty} \frac{1}{(2 n-1)^{2}} \sin \left(\frac{(2 n-1) \pi}{2}\right) E_{2 n-1} .
$$

(c) the concentration difference between top and bottom of the cell:

$$
\frac{\Delta c(t)}{\Delta c^{\mathrm{st}}}=1-\frac{8}{\pi^{2}} \sum_{n=1}^{\infty} \frac{1}{(2 n-1)^{2}} E_{2 n-1}
$$

Each of the above approaches provides both Soret $S_{T} \propto \Delta c^{\text {st }}$ and diffusion $D$ coefficients, but the amount of data involved in the extraction of coefficients is essentially different. Indeed, the gradient approach (b) implicitly assigns more weight to the data points located at the center of the cell, while the concentration difference (c) gives greater weight to the data points near the horizontal walls. Thus, the application of different solutions can also lead to slightly different results.

\section{Fitting procedures}

The input data for fitting are obtained from refractive index variations during either Soret or diffusion step over the experimental time. As can be seen from Fig. 7, the curves for $n_{1}^{\prime}$ and $n_{2}^{\prime}$ obtained at different wavelengths 
are clearly separated from each other. The analytical solution for the heat and mass transfer problem describing the Soret separation in ternary mixtures, $c_{i}^{\prime}(z, t)$, comprise six unknown quantities, four diffusion and two thermodiffusion coefficients. The task of fitting procedure is to determine the transport coefficients at which the equality in Eqs. (5)-(6) is fulfilled.

The fitting with six unknown is very delicate problem and its detail description can found in the Supplementary to this paper. Here we underline that, as a rule, six parameter fitting is extremely unstable. Furthermore, recently was shown that, only five out of the six unknown quantities can be obtained with a satisfactory accuracy in a two-color optical Soret experiment on ternary mixtures ${ }^{32}$. Using the specific combination of unknown quantities in analytical solution, the problem can we transferred to four-parameter fit ${ }^{26}$ or, to twoparameter fit, assuming that two eigenvalues are equal ${ }^{18}$. Details of these fitting procedures also can be found in the Supplementary to the paper.

Recall that despite on the fitting procedure, the solution of the system of linear equation Eqs. (5)-(6) is a challenging task because the condition number of the matrix of optical contrasts $\mathbf{R}$ is rather high, $\mathcal{K}=80$.

\section{Correction considering non-linear fields}

The temperature field in the DCMIX cell is spatially non-linear due to the cell design ${ }^{16}$, see also Fig. 3. It causes the non-linearity of the refractive index distribution, and, consequently, a concentration field obtained via the refractive index variation exhibits similar nonlinearity. However, the working equations describing the problem are obtained for the linear concentration field (one-dimensional). Hence, optical integration of a deformed field leads to an underestimated value of the Soret separation.

The different suggestions for the correction of data are discussed in detail at the Supplementary material to this paper. Here we just note, that the most successful approach is based on tomography reconstruction of the concentration field ${ }^{10,18,19}$. Indeed, it was demonstrated that the tomography-reconstructed $\Delta c^{\mathrm{st}}$ is $10-15 \%$ larger than its raw value.

\section{B. Analysis of processing approaches}

As can be seen from Sect. III A, a variety of combinations to create an integral processing scheme, starting from the optical phase extraction and ending by quantitative information on mass transport coefficients, is really wide. Testing of all possible combinations is a huge and somewhat excessive task. For the present work, we have selected seven processing schemes (listed in Table VI) that allow to obtain multiple combinations of pairs, in which only one processing step is different.
TABLE VI. List of all tested data processing schemes and an average separation provided by each scheme. The separation obtained by P_1 is taken as unity.

\begin{tabular}{lcccccc}
\hline $\begin{array}{l}\text { Proc. } \\
\text { ID }\end{array}$ & Phase $^{\text {a }}$ Step $^{\mathrm{b}}$ & $\begin{array}{c}\text { Solu- } \\
\text { tion }^{\mathrm{c}}\end{array}$ & Fit $^{\mathrm{d}}$ & $\begin{array}{c}\text { Correc- }^{\text {tion }}{ }^{\mathrm{e}} \\
\text { P_1 }\end{array}$ & $\begin{array}{c}\text { Average } \\
\text { separation }\end{array}$ \\
\hline PS & S & F & 2 & - & 1 \\
P_2 & PS & S & G & 2 & - & 0.950 \\
P_3 & PS & S & F & 2 & T & 1.152 \\
P_4 & FT & S & F & 2 & - & 0.994 \\
P_5 & FT & D & F & 2 & - & 0.975 \\
P_6 & FT & S & F & 4 & - & 1.179 \\
P_7 & FT & D & F & 4 & - & 1.016 \\
\hline
\end{tabular}

a 'PS' stands for the phase-shift, and 'FT' for the Fourier transform for the optical phase extraction.

b 'S' stands for the Soret step, and 'D' for the diffusion step.

d ' 4 ' stands for four-parameter fit and '2' for two-parameter one.

c ' $F$ ' stands for the full-path, and 'G' for the gradient solution.

e ' $T$ ' stands for the data correction made by tomography.

Note that we have processed all the runs listed in Table $\mathrm{V}$ by each of the schemes presented in Table VI to get a statistically valuable output. Thus, the value of $\Delta n / \Delta T$ in the last column of the Table VI is the mean value over the all nine runs. Then, we have normalized this average quantities by the value in the first raw, which is taken equal to unity. This suggests the more transparent comparison.

For example, comparison of the processing schemes:

- P_1 and P_4 reveal the difference due to the optical phase extraction, i.e., phase shift and Fourier transform. - P_4 vs. P_5 and P_6 vs. P_7 indicate the difference between results found from Soret or diffusion steps of experiments.

- P_1 and P_2 compare the results for the full-path and gradient solutions.

- P_4 vs. P_6 and P_5 vs. P_7 display the difference between four- and two-parameter fits.

- P_1 and P_3 quantify the effect of the tomography correction.

\section{RESULTS AND DISCUSSION}

\section{A. Selection of the most reliable processing scheme}

In the previous section (Sec. III A) we have analysed all the possible varieties of processing schemes. To select the most reliable processing schemes, we need to establish a criterion to conclude that some schemes are superior to others. It was mentioned already that the optically measured separation in the SODI-DCMIX experimental cells is undervalued due to cells design ${ }^{18,33}$. Thus, we suggest the schemes providing a higher value of the component separation (statistically defined over all the runs) to be considered as preferable. According to this criterion, two 
TABLE VII. The differences of refractive indices in steady state between differentially heated walls per degree of temperature difference $\left(-\Delta n^{\text {st }} / \Delta T\right) / 10^{-4} \mathrm{~K}^{-1}$ measured onboard the ISS in the cell \#1 at two wavelengths. ULB indicates the result obtained by Belgian team, and RAS - by Russian team, respectively.

\begin{tabular}{lccccc}
\hline Run & $T_{0}$ & \multicolumn{2}{r}{ P_3 (ULB) } & \multicolumn{2}{c}{ P_6 (RAS) } \\
\cline { 3 - 6 } & $(\mathrm{K})$ & $670 \mathrm{~nm}$ & $935 \mathrm{~nm}$ & $670 \mathrm{~nm}$ & $935 \mathrm{~nm}$ \\
\hline Run_01 & 298.15 & 1.3850 & 1.3610 & 1.4685 & 1.4329 \\
Run_06 & 298.15 & 1.4397 & 1.4136 & 1.4592 & 1.4202 \\
Run_01bis & 298.15 & 1.3886 & 1.3634 & 1.4402 & 1.4082 \\
Run_11 & 301.15 & 1.1890 & 1.1672 & 1.2115 & 1.1827 \\
Run_16 & 304.15 & 0.9640 & 0.9512 & 0.9883 & 1.0079 \\
Run_21 & 298.15 & 1.3984 & 1.3752 & 1.4530 & 1.4207 \\
Run_26 & 295.15 & 1.6214 & 1.5926 & 1.6817 & 1.6552 \\
Run_32 & 304.15 & 0.9674 & 0.9506 & 0.9498 & 0.9383 \\
Run_33 & 306.15 & 0.8238 & 0.8126 & 0.8343 & 0.7865 \\
\hline
\end{tabular}

processing schemes, P_3 and P_6, are selected as superior. They do not provide identical results, but the difference between them in $\Delta n^{\text {st }}$ is only $2.3 \%$ in average. The separation values obtained by these best schemes will be then used for the extraction of Soret coefficients of the DCMIX $_{2}$ ternary mixture and for an estimation of their error-bars.

\section{B. Soret coefficients determined by the best processing schemes}

The differences of refractive indices in steady state between the hot and cold walls, $\Delta n^{\text {st }}$, were determined in the course of the fitting over time interval equal to the duration of the experiment.

Taking into account Eqs.(5)-(6) and (3), the difference of refractive indices between differently heated walls at steady state is written as

$$
\left(\begin{array}{c}
\Delta n_{1}^{s t} \\
\Delta n_{2}^{s t}
\end{array}\right)=-\Delta T\left[\begin{array}{ll}
R_{11} & R_{12} \\
R_{21} & R_{22}
\end{array}\right]\left(\begin{array}{c}
S_{T, 1}^{\prime} \\
S_{T, 2}^{\prime}
\end{array}\right)
$$

It follows that the difference of refractive indices $\Delta n_{i}^{s t}$ caused by the Soret effect is proportional to the imposed temperature difference $\Delta T$. To unify and compare the experiments at different $\Delta T$, the values of $\Delta n_{i}^{s t}$ per Kelvin, i.e. the ratio $\Delta n_{i}^{s t} / \Delta T$, will be used. In what follows, we refer to this value as the optical separation. These values, obtained by the best selected scheme of processing, P_3 and P_6, are given in Table VII for nine experimental runs conducted at various mean temperatures. The tabular values of the optical separations are then used for the calculation of Soret coefficients $S_{T, i}^{\prime}$ by solving the system of linear equations (14). The elements $R_{i j}$ of the matrix of contrast factors, required for the calculation, are given in Table IV.

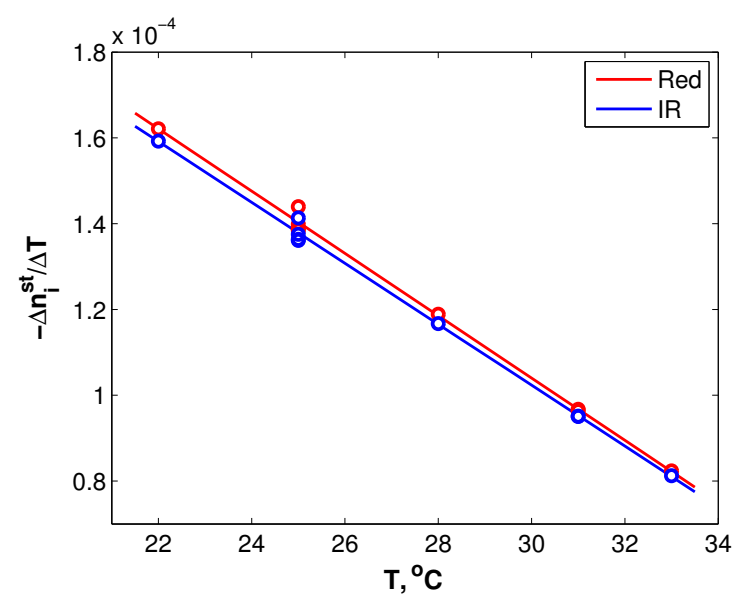

FIG. 8. Temperature dependence of the optical separation $\left(-\Delta n^{\text {st }} / \Delta T\right)$ measured onboard the ISS in the cell \#1 at two wavelengths, $\lambda=670 \mathrm{~nm}$ (the red curve) and $\lambda=935 \mathrm{~nm}$ (the blue curve) (data processed by P_3 of ULB).

The temperature dependence of the optical separations obtained by the scheme P_3 is shown in Fig. 8, using data of Table VII. The important outcome that follows from the figure is the perfectly linear temperature dependence of $\Delta n_{i}^{s t} / \Delta T$. This observation, reported here for the first time for a ternary mixture, reveals similarity with binary mixtures. Indeed, the temperature dependence of the Soret separation (or Soret coefficient) in binary mixtures does follow a linear trend. As examples, we may refer to the extended study of water-ethanol mixtures ${ }^{28}$ and the binary mixture from DCMIX $_{2}$ cell array ${ }^{19}$.

Another interesting observation from figure Fig. 8 is that the behaviour of curves for different wavelengths is somewhat similar, which leads to conclude on the constancy of the ratio $\Delta n_{1}^{\text {st }} / \Delta n_{2}^{\text {st }}$ over the examined temperature range. This finding also was confirmed by the all data listed in Table VII. Further, we have determined this ratio for the nine experimental runs using seven different processing schemes indicated in the Table VI. The statistical distribution of the ratio $\Delta n_{1}^{\text {st }} / \Delta n_{2}^{\text {st }}$ for these 63 values is shown in Fig. 9.

Obviously, there is a dense cluster of results with practically the same value of the ratio $\Delta n_{1}^{\mathrm{st}} / \Delta n_{2}^{\mathrm{st}}=$ $1.019 \ldots 1.020$, and rather limited scattering of the results around this value. An interesting practical consequence is that it offers another criterion for the selection of the best processing scheme or at least for the elimination of unreliable ones. We can note from Fig. 9, that three of the tests yielded data that clearly deviates from the common trend, indicating some problems in the data extraction.

Keeping in mind that the ratio of two refractive index differences is temperature independent over the considered interval of the mean temperatures, we can re-write 


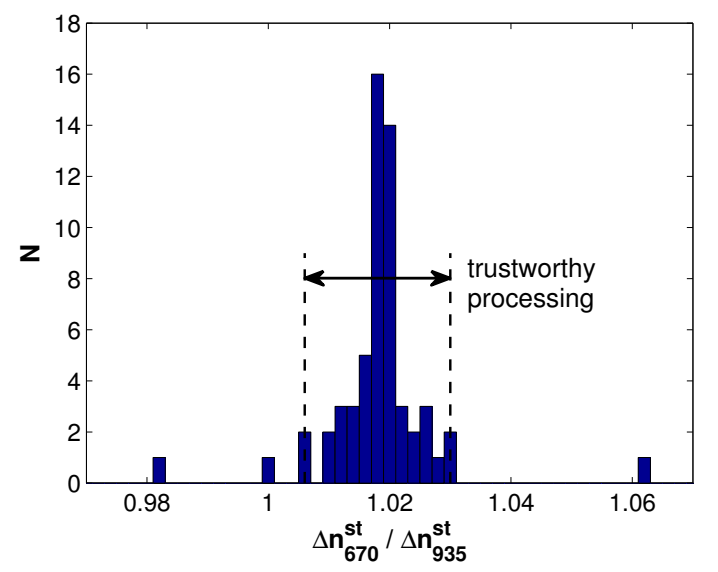

FIG. 9. Histogram showing the number $(N)$ in the set of 63 values calculated for $\Delta n_{1}^{\text {st }} / \Delta n_{2}^{\text {st }}$; this ratio was determined for nine expeimental runs from Table VII using the seven processing schemes indicated in Table VI.

Eq. (14) in the form

$$
\left(\begin{array}{c}
S_{T, 1}^{\prime} \\
S_{T, 2}^{\prime}
\end{array}\right)=-\frac{\Delta n_{2}^{s t}}{\Delta T}\left[\begin{array}{ll}
R_{11} & R_{12} \\
R_{21} & R_{22}
\end{array}\right]^{-1}\left(\begin{array}{c}
\frac{\Delta n_{1}^{s t}}{\Delta n_{2}^{s t}} \\
1
\end{array}\right)
$$

Assuming that the temperature dependence of the contrast factors $R_{i j}$ is very weak, the only factor that is not constant is $-\Delta n_{2}^{\text {st }} / \Delta T$. Furthermore, it means that the temperature dependence affects the Soret coefficients in the same way as the scaling factor $-\Delta n_{2}^{\text {st }} / \Delta T$, which has a linear temperature dependency (see Fig. 8).

Thus, we can draw two conclusions: first, both Soret coefficients vary linearly with temperature; second, they are proportional to each other.

Once we have transformed the optical separations presented in Table VII into Soret coefficients using Eq. (15), we plot them in the parameter space of two Soret coefficients as shown in Fig. 10. Presenting data in this way is convenient, since each pair of Soret coefficients relating to the examined mean temperature of the ternary mixture is represented by a single point on the graph. The distribution of points on the graph appears to be stochastic, and a simple visual observation does not allow to confirm the existence of proportionality between the two coefficients. To illustrate the proportionality we need deeper insight into data in Table VII.

Let us implement a linear interpolation, which provides more accurate values of $-\Delta n_{i}^{\text {st }} / \Delta T$ for a set of five examined temperatures compared to the individual measurements. The interpolation was performed independently for the data of each column of Table VII. Then, the values of the optical separation obtained by the interpolation were used in calculation of the Soret coefficients. The coefficients obtained in this way are given in Table VIII for each type of processing and as merged values obtained as a result of simple averaging.

Another important problem leading to a scattering of

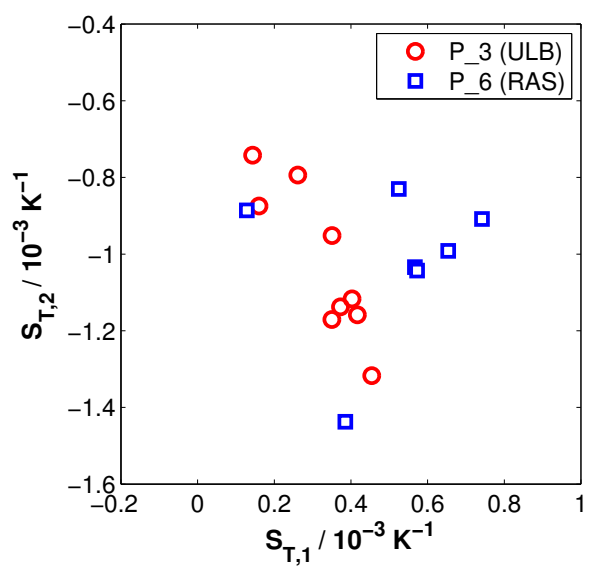

FIG. 10. The Soret coefficients of the ternary mixture toluenemethanol-cyclohexane directly calculated from the normalized difference of the refractive indices given in Table VII using Eq. 15. Data corresponds to microgravity experiments at different mean temperatures.

TABLE VIII. Soret coefficients $S_{T, i} / 10^{-3} \mathrm{~K}^{-1}$ of the ternary mixture toluene-methanol-cyclohexane determined from the optical separation with subsequent linear interpolation.

\begin{tabular}{lcccccc}
\hline$T_{0}$ & \multicolumn{2}{c}{ P_3 (ULB) } & \multicolumn{2}{r}{ P_6 (RAS) } & \multicolumn{2}{c}{ average } \\
\cline { 2 - 7 }$(\mathrm{K})$ & $S_{T, 1}^{\prime}$ & $S_{T, 2}^{\prime}$ & $S_{T, 1}^{\prime}$ & $S_{T, 2}^{\prime}$ & $S_{T, 1}^{\prime}$ & $S_{T, 2}^{\prime}$ \\
\hline 295.15 & 0.472 & -1.303 & 0.589 & -1.275 & 0.530 & -1.289 \\
298.15 & 0.387 & -1.145 & 0.504 & -1.098 & 0.445 & -1.122 \\
301.15 & 0.301 & -0.988 & 0.420 & -0.921 & 0.361 & -0.955 \\
304.15 & 0.216 & -0.831 & 0.336 & -0.744 & 0.276 & -0.788 \\
306.15 & 0.160 & -0.727 & 0.280 & -0.626 & 0.220 & -0.676 \\
\hline
\end{tabular}

the Soret coefficients in DCMIX ternary mixtures is that the matrix of contrast factors is not well-conditioned ${ }^{25}$. This can be seen from the condition number for the considered mixture which is $\mathcal{K}=80$. The effect of the illconditioning is a notable increase in the error on the calculated Soret coefficients originating from a minor discrepancy between the measured optical separations in two tests. This error amplification was confirmed in the benchmark on a ternary mixture of THN-IBB$\mathrm{nC} 12^{18,26,34}$.

The error on the Soret coefficients has a remarkable property: instead of forming a radially uniform scattering cloud around the solution point $\left\{S_{T, 1}, S_{T, 2}\right\}$, it forms a very elongated ellipsoid practically degenerated into a line, as shown in Fig. 11. The orientation of the line depends on properties of the matrix of contrast factors, and may differ for different mixtures.

We shall demonstrate the important consequence of this error property. Two solutions originating from two experiments with almost identical measured $\left\{\Delta n_{1}^{\text {st }}, \Delta n_{2}^{\text {st }}\right\}$ can be located rather far from each other in the space of Soret coefficients. However, they belong to the same line of error-bar defined by the matrix of 


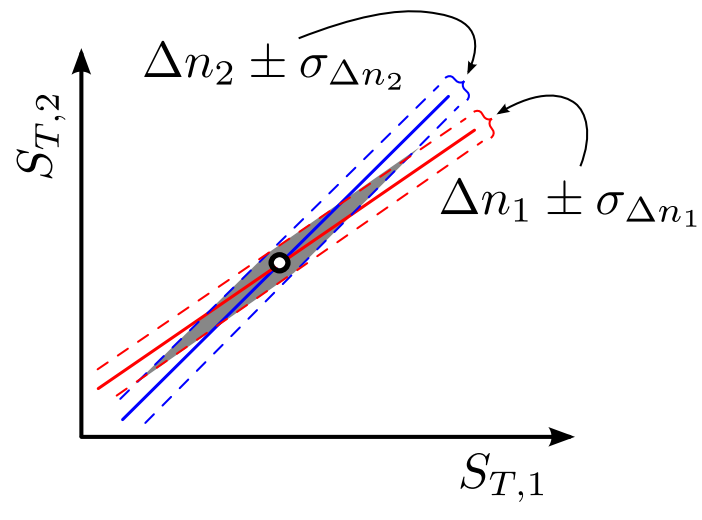

FIG. 11. The illustration of the error propagation of the Soret coefficients calculated with Monte-Carlo approach with an added noise. The blue and red solid lines are isolines which correspond to experimentally measured $\Delta n_{i}$ which give a solution shown by the open circle. Grey area covers a locus of possible scattering of this solution

contrast factors.

For the sake of an example test, two close sets of data for $\Delta n_{i}^{\text {st }} / \Delta T$ were selected as follows: one was taken directly from Table VII and the other was obtained after the linear interpolation of the same data. Then, for both sets, the Soret coefficients $S_{T, i}^{\prime}$ were calculated by the use of Eqs. (15). The Soret coefficients obtained on the basis of original data from Table VII are shown by open circles in Fig. 12 while the Soret coefficients calculated from the interpolated $\Delta n_{i}^{\text {st }} / \Delta T$ are shown by filled circles.

Then, we determined the above-mentioned error-bars applying the Monte-Carlo approach. The normally distributed random noise was added to the interpolated optical separations $\Delta n_{i}^{\text {st }} / \Delta T$. The standard deviation for the noise simulation has been taken as one-third of the absolute average deviation of the experimental points from the interpolation line, which was found to be $0.7 \%$ for the processing scheme P_3 and 1.1\% for P_6. The Soret coefficients were calculated from these noisy data using Eqs. (15). The possible scattering of the Soret coefficients obtained by Monte-Carlo simulation is shown in Fig. 12 by blue and red dots, actually, they form lines.

This merged figure clarifies that the points that seemed to be randomly dispersed in the space of Soret coefficients are very well structured in reality. All the measurements at the same mean temperature do strictly belong to corresponding error-bars. As the final value of the Soret coefficients, we propose to use the average value obtained by the two processing schemes, i.e., P_3 and P_6. These values are indicated in Fig. 12 by the green rhombi.

Note that the merged Soret coefficients can be obtained in another way. Instead of a linear interpolation of each column in Table VII, the interpolation can be applied for a larger data set combining the values of $\Delta n^{s t} / \Delta T$ obtained by two schemes, P_3 and P_6. As a result, we get a pair of linear dependences of the optical separations

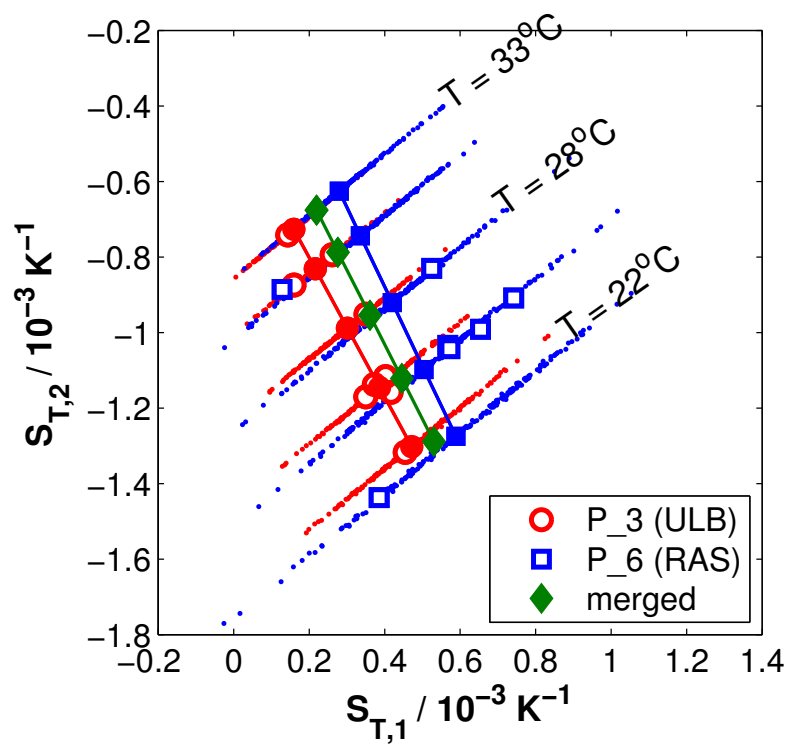

FIG. 12. Comparison of ternary Soret coefficients calculated from measured separations (open circles), determined from linear trend for optical separations (closed circles) and their possible scattering due to an error of separation obtained by Monte-Carlo simulation (dots). Closed green circles present final merged values

at working wavelengths $\lambda=670 \mathrm{~nm}$ and $935 \mathrm{~nm}$, and then transform them into a set of Soret coefficients. It appears that both approaches provide an identical result.

To finish, let us discuss the obtained results summarized in Fig. 12. The Soret coefficient $S_{T, 1}^{\prime}$ of the denser component, toluene, is positive and its value varies in the range between $0.2 \times 10^{-3} \mathrm{~K}^{-1}$ and $0.6 \times 10^{-3} \mathrm{~K}^{-1}$. The Soret coefficient $S_{T, 2}^{\prime}$ for methanol is negative and by absolute value is always larger than $S_{T, 1}^{\prime}$. However, the solutal expansion of methanol is much smaller than toluene. Then, the separation ratios $^{35}$

$$
\Psi_{i}=-\left(\beta_{c} / \beta_{T}\right) S_{T, i}^{\prime}
$$

have similar values but different signs, for example, $\Psi_{1}=$ -0.397 and $\Psi_{2}=0.343$ at $T_{\text {mean }}=298.15 \mathrm{~K}$. Thus, this system has intriguing stability properties and is highly interesting example for theoretical analysis.

\section{v. CONCLUSIONS}

We report results of the measurements of Soret coefficients in the ternary mixture toluene-methanolcyclohexane with composition $0.62 / 0.31 / 0.07$ in mass fractions. The experiments were conducted in microgravity environment onboard the ISS.

On a qualitative level, we have established that Soret coefficients present a linear dependence on the mean temperature. Such a finding is reported for the first time on ternary mixtures. 
TABLE IX. Soret coefficients $S_{T, i} / 10^{-3} \mathrm{~K}^{-1}$ of the ternary mixture toluene-methanol-cyclohexane (0.62-31$0.07) \mathrm{kg} \mathrm{kg}^{-1}$.

\begin{tabular}{lccc}
\hline$(\mathrm{K})$ & toluene & methanol & cyclohexane \\
\hline 295.15 & 0.530 & -1.289 & 0.759 \\
298.15 & 0.445 & -1.122 & 0.677 \\
301.15 & 0.361 & -0.955 & 0.634 \\
304.15 & 0.276 & -0.788 & 0.512 \\
306.15 & 0.220 & -0.676 & 0.456 \\
\hline
\end{tabular}

On a quantitative level (see Table IX), we have obtained that for all the mean temperatures under consideration, the Soret coefficient of the denser component is small and positive, and for the second one is large and negative. This puzzling combination of the Soret coefficients provides ground for comprehensive theoretical studies of double-diffusive instabilities.

Except for the proper result measurements, the present work also presents an extensive study of possible methodologies to process raw data from the Soret experiments in ternary mixtures. Analysis of raw data from the previous and current orbital DCMIX experiments has established that data processing is much more complex than originally thought. It consists of five major steps and each of them can be treated in a few different ways.

The nine experimental runs in cell \#1 of DCMIX $_{2}$ experiment were processed by seven schemes, and two of the schemes were selected as the most reliable. Although the variation of the refractive indices obtained by these schemes differs by about $2 \%$, the obtained Soret coefficients display larger scattering. We investigate the dynamics of the error propagation and show that the obtained Soret coefficients are well structured. The error bar of the Soret experiment forms a very elongated ellipsoid instead of forming a radially uniform cloud around the solution. All Soret coefficients measured at the same mean temperature and processed by the two selected schemes are located on the same line of the error-bar.

This work highlights the subtle dependence of the obtained Soret coefficients on the processing scheme. The two selected schemes are recommended for the processing the results in the remaining four cells of the $\mathrm{DCMIX}_{2}$, as well as for ground-based experiments.

\section{ACKNOWLEDGEMENTS}

This work has been supported by the PRODEX programme of the Belgian Federal Science Policy Office. This work has been developed in the framework of the cooperative project DCMIX (No. AO-2009-0858/1056) of the European Space Agency and the Russian Space Agency (Roscosmos). The authors (ULB) are indebted to Prof. J.C. Legros (ULB, Brussels) for valuable discussions.

${ }^{1}$ K. Ghorayeb, A. Firoozabadi, T. Anraku, SPEJ 8, 114 (2003)

${ }^{2}$ G. Galliero, H. Bataller, F. Croccolo, R. Vermorel, P.A. Artola, B. Rousseau, V. Vesovic, M. Bou-Ali, J.M. Ortiz de Zárate, S. Xu et al., Microgravity Sci. Tec. 28, 79 (2016)

${ }^{3}$ S. Duhr, D. Braun, Proc. Natl. Acad. Sci. USA 103, 19678 (2006)

${ }^{4}$ W. Köhler, K. A., W. Zimmermann, Adv. Polym. Sci. 227, 145 (2010)

${ }^{5}$ W. Köhler, K. Morozov, J. Non-Equilib. Thermodyn. 41, 151 (2016)

${ }^{6}$ J.K. Platten, ASME. J. Appl. Mech 73, 5 (2005)

${ }^{7}$ A. Königer, H. Wunderlich, W. Köhler, J. Chem. Phys. 132, $174506(2010)$

${ }^{8}$ P. Blanco, M. Bou-Ali, J.K. Platten, D.A. de Mezquia, J.A. Madariaga, C. Santamara, J. Chem. Phys. 132, 114506 (2010)

${ }^{9}$ A. Mialdun, V. Shevtsova, J. Chem. Phys. 138, 161102 (2013)

${ }^{10}$ V. Shevtsova, Y. Gaponenko, V. Sechenyh, D. Melnikov, T. Lyubimova, A. Mialdun, J. Fluid Mech. 767, 290 (2015)

${ }^{11}$ M. Bou-Ali, A. Ahadi, D. Alonso de Mezquia, Q. Galand, M. Gebhardt, O. Khlybov, W. Köhler, M. Larrañaga, J. Legros, T. Lyubimova et al., Eur. Phys. J. E 38, 30 (2015)

${ }^{12}$ A. Martin, M.M. Bou-Ali, Comptes Rendus Mcanique 339, 329 (2011)

${ }^{13}$ H. Bataller, T. Triller, B. Pur, W. Khler, J.M.O. de Zrate, F. Croccolo, Eur. Phys. J. E 40, 35 (2017)

${ }^{14}$ V. Shevtsova, C. Santos, V. Sechenyh, J.C. Legros, A. Mialdun, Microgravity Sci. Tec. 25, 275 (2014)

${ }^{15}$ C. Santos, A. Mialdun, J. Legros, V. Shevtsova, J. Appl. Polym. Sci. 133, 43449 (2016)

${ }^{16}$ A. Mialdun, C. Minetti, Y. Gaponenko, V. Shevtsova, F. Dubois, Microgravity Sci. Tec. 25, 83 (2013)

${ }^{17}$ A. Ahadi, S.V. Varenbergh, M.Z. Saghir, J. Chem. Phys. 138, 204201 ( 17) (2013)

${ }^{18}$ A. Mialdun, J.C. Legros, V. Yasnou, V. Sechenyh, V. Shevtsova, Eur. Phys. J. E 38, 27 (2015)

${ }^{19}$ A. Mialdun, V. Shevtsova, J. Chem. Phys. 143, 224902 (2015)

${ }^{20}$ E. Lapeira, M. Gebhardt, T. Triller, A. Mialdun, W. Köhler, V. Shevtsova, M.M. Bou-Ali, J. Chem. Phys. 146, 094507 (2017)

${ }^{21}$ E. Lapeira, Ph.D. thesis, Mondragon Unibertsitatea, Mondragon (2017), departamento de Mecnica y Produccin Industrial

${ }^{22}$ M. Hendrix, A. Leipertz, M. Fiebig, G. Simonsohn, International Journal of Heat and Mass Transfer 30, 333 (1987)

${ }^{23}$ T. Grossmann, J. Winkelmann, J. Chem. Eng. Data 54, 405 (2009)

${ }^{24}$ V. Sechenyh, J.C. Legros, V. Shevtsova, Journal of Chemical \& Engineering Data 57, 1036 (2012)

${ }^{25}$ V. Shevtsova, V. Sechenyh, A. Nepomnyashchy, J. Legros, Philos. Mag. 91, 3498 (2011)

${ }^{26}$ O. Khlybov, I. Ryzhkov, T. Lyubimova, Eur. Phys. J. E 38, 29 (2015)

${ }^{27}$ R. Taylor, R. Krishna, Multicomponent Mass Transfer, Wiley Series in Chemical Engineering (Wiley, 1993), ISBN 9780471574170

${ }^{28}$ A. Königer, B. Meier, W. Köhler, Philos. Mag. 89, 907 (2009)

${ }^{29}$ J.F. Torres, A. Komiya, D. Henry, S. Maruyama, J. Chem. Phys. 139, 074203 (2013)

${ }^{30}$ M. Gebhardt, W. Köhler, A. Mialdun, V. Yasnou, V. Shevtsova, J. Chem. Phys. 138, 114503 (2013)

${ }^{31}$ A. Mialdun, V. Shevtsova, J. Chem. Phys. 134, 044524 (2011)

${ }^{32}$ M. Gebhardt, W. Köhler, J. Chem. Phys. 142, 084506 (2015)

${ }^{33}$ A. Mialdun, V. Shevtsova, Appl. Opt. 56, 572 (2017)

${ }^{34}$ M. Gebhardt, W. Köhler, Eur. Phys. J. E 38, 24 (2015)

${ }^{35}$ I.I. Ryzhkov, V.M. Shevtsova, Physics of Fluids 19, 027101 (2007) 